Andrew J. Larner

Received: 5 April 2005

Accepted in revised form: 12 May 2005

Published online: 1 August 2005

A.J. Larner (凶)

Walton Centre for Neurology and

Neurosurgery,

Lower Lane, Fazakerley,

Liverpool L9 7LJ, UK

e-mail: a.larner@thewaltoncentre.nhs.uk

Tel.: +44-151-529-5727

Fax: +44-151-529-5513

\title{
Guidelines for the management of headache in primary care: are they being used?
}

\author{
Abstract The uptake of guidelines \\ for the management of headache \\ in primary care was assessed using \\ surrogate measures in patients \\ with headache referred \\ to secondary care (number of prior \\ general practitioner attendances \\ and medications prescribed). \\ The results suggest that headache \\ guidelines are seldom, if ever, \\ applied in this geographical \\ location.
}

Key words Guidelines • Headache • Migraine
Two sets of guidelines on management of primary headache disorders (PHD) in the primary care setting have been published in the UK in the past five years [1-3]. These suggest that most PHD may be diagnosed, treated and followed-up in primary care, and advocate stepped or stratified approaches which, if not immediately successful, inevitably involve $>1$ general practitioner (GP) visit or drug prescription. The aim of this study was to ascertain how often headache guidelines are used in primary care.

Consecutive new referrals complaining of headache, seen at three general adult neurology clinics in north-west England, were asked how often they had visited their GP prior to referral, and how many different medications were prescribed for headache. Neurologist diagnosis of headache syndromes was based upon internationally agreed criteria [4].

Over a 6-month period (mid April - mid October 2004), 114 of 475 new referrals had headache, 105 referred by GP (92\%; $95 \%$ confidence interval (CI) 87\%-97\%; Table 1). Twenty-two reported only one GP
Table 1 Demographic and diagnostic data

\begin{tabular}{ll}
\hline & $\begin{array}{l}\text { GP-referred } \\
\text { headache patients }\end{array}$ \\
\hline$n$ & 105 \\
Age range, years & $17-78$ \\
Median age, years & 37 \\
Sex ratio M:F (male \%) & $40: 65(38 \%)$ \\
Specific diagnosis & \\
$\quad$ CTTH & 55 \\
$\quad$ FTTH & 4 \\
$\quad$ MO & 12 \\
$\quad$ MA & 11 \\
$\quad$ CM/TM & 18 \\
$\quad$ MOH & 4 \\
CH/TAC & 1
\end{tabular}

$C T T H$, chronic tension-type headache; $F T T H$, frequent tension-type headache; $M O$, migraine without aura; $M A$, migraine with aura; $C M / T M$, chronic migraine/transformed migraine; $M O H$, medication overuse headache; $C H / T A C$, cluster headache/trigeminal autonomic cephalalgia 
visit prior to referral $(21 \%$; $95 \%$ CI $13 \%-29 \%)$, a result similar to a previous study $(17 \% ; 95 \%$ CI $10 \%-24 \%)$ [5], suggesting this does accurately reflect local primary care practice. Of those reporting $>1$ GP consultation, 34 reported $\leq 1$ medication prescribed for headache prior to referral (32\%; 95\% CI 23\%-41\%). Therefore, the total proportion of GP headache referrals seen only once and/or prescribed only one medication was $53 \%$ (95\% CI $43 \%-63 \%)$. Hence, using this surrogate measure of guideline adoption, the most optimistic interpretation is that $47 \%$ of referred patients were managed using PHD guidelines, although no referral specifically mentioned their use.

Reasons for failure to adopt guidelines include inadequate awareness and time constraints. Because guidelines are needed to set recognisable and acceptable standards of good practice, and develop a common understanding, their adoption in primary care should be encouraged.

Acknowledgement Thanks are due to Dr C.A.H. Fisher for advice.

\section{References}

1. British Association for the Study of Headache (2000) Review of the organisation of headache services in primary care and recommendations for change. British Association for the Study of Headache, London [www.bash.org.uk]
2. Dowson AJ, Lipscombe S, Sender J, Rees T, Watson D on behalf of the MIPCA Migraine Guidelines Development Group (2002) New guidelines for the management of migraine in primary care. Curr Med Opin Res 18:414-439 [www.mipca.org.uk]

3. Guidelines: summarising clinical guidelines for primary care (2004) 23:139-148 [www.eguidelines.co.uk]
4. International Headache Society Classification Subcommittee (2004) The international classification of headache disorders, 2 nd edn. Cephalalgia 24[Suppl 1]:1-160

5. Gahir KK, Larner AJ (2004) What role do community pharmacists currently play in the management of headache? A hospital-based perspective. Int J Clin Pract 58:257-259 